Numenius arquatus hielt sich diesen Herbst in grossen Schaaren auf unsern und den Aengern und Wiesen an der Elbe auf. Die Vögel sind ungemein aufmerksam und scheu. Vergebens bemühten sich Gegner, einen zu erlegen.

Ciconia alba nahm seinen Horst auf einer Schener am Ende des Dorfes ein, tödtete aber vor seinem Abzuge die fast flüggen Jungen. Wahrscheinlich ist er derselbe mordlustige Storch, der vor einigen Jahren sein Weib tödtete und sich den andern Tag ein frisches nahm.

Sterna hirundo und minuta wohnen seit einigen Jahren auf einem Kieshäger der Elbe, nahe der Rosslau-Dessauer Brücke. Der Häger hat sich erst seit Jahren gebildet. Bei dem diesjährigen niedern Wasserstande der Elbe wurde es Knaben möglich, hinüber zu waten und die Eier zu zertrümmern. Gleichwohl haben die Vögel, ebenso wie die Flussregenpfeifer, ihr Gebiet behauptet. Die Arten sind daran gewöhnt, dass Menschen und Krähen ihnen die Eier rauben.

Mühlstedt, im November 1864 .

\title{
Notizen über den Vogelzug im Herbst 1864, sowie die ornitho- logischen Vorkommnisse in den Ländern der Bischárin, Omáráb and Hadendoa zwischen Berber und Sanakin.
}

\section{Von \\ Hofrath Dr. Th. v. Henglin.}

Es war mir gegen Ende meines letzten Aufenthalts am Nil in Berber (August 1864) sehr auffallend, in wenigen Tagen und verhältnissmässig sehr zeitig eine grosse Menge geflügelter Wanderer ans Europa ankommen zu sehen. Ob ausserordentliche meteorologische Erscheinungen im Norden hiermit in Verbindung stehen, kann ich natürlich von hier aus nicht beurtheilen, aber ich glaube, dass die zuerst hier erscheinenden Vorboten des Winters gewöhnlich südlichere Bewohner sind, die auch während der kalten Jahreszeit vielleicht weiter nach Süden vorgehen, als ihre in kältern, nordischen Regionen acclimatisirten Geschwister; denn die ersten Zugvögel, selbst von Arten, die gevöhnt sind in grössern Gesellschaften zu wandern, erscheinen meist Anfangs einzelner.

Am 26. August sah ich am Nil die érsten Upupa. Ardea atricollis und bubulcus brütet um diese Zeit noch. 
Vogelzug sowie ornitholog. Vorkommnisse in N.-O.-Africa. 43

Am 27. August bis 1. September erschienen um Berber: Ibis falcinella, Aedon galactodes, Cypselus murarius, Lanius spinitorquus, L. leucometopon, Coracias garrula, Muscicapa grisola? Circus pallidus, Sylvia hypolais, Merops aegyptius.

Am 10. September bemerkte ich in der Wüste der Bischarin zwischen Berber und dem Abadab-Gebirge: Oriolus galbula zahlreich, Motacilla cinereocapilla und melanocephala, am 12. September Coturnix, 13. Apr. Aquila pennata und Circaëtos brachydactylus, Muscicapa grisola, am 14. Sylvia (Ruticilla) tithys, Lanius collurio, Saxicola melanura (vielleicht hier Standvogel), Alauda brachydactyla in Flügen, Merops albicollis, Sylvia garrula, Sylvia Rüppelli; am 15 bis 20. September in den Gebirgen zwischen Abadab und dem rothen Meer: Luscinia minor, Sylvia suecica, Machetes pugnax, Hirundo rustica, Ruticilla phoenicura, Emberiza caesia. Am rothen Meer selbst den 27. Septbr.: Gallinula porzana und Caprimulgus europaeus.

Zwischen dem Abadab- und Abu-Goloda-Gebirge sah ich mehrere Flüge von Turteltauben, vielleicht wandernde Columba turtur.

Die Fauna im Allgemeinen ist in den von mir jetzt bereisten Bedja-Gebirgen bis gegen das obere Nilthal hin zwar ziemlich vielfältig, aber nicht reich an Individuenzahl, obgleich namentlich in den östlichen Gebirgsgegenden kein Mangel an Lebensbedingungen ist.

Die erste Station meiner Reise rom Nil zum rothen Meer ist der nur 5 Meilen vom Nil entfernte Wüstenbronnen Moha Bey in ciner mit Gramineen, Mimosen- und Tundubbüschen bedeckten Niederung. Von Standvögeln sah ich hier, ausser Cathartes percnopterus und Milanen, Corvus umbrinus (häufig bis in die Gebirge bei Sanakin, aber nicht am Meer), Certhialauda bifasciata (mehr in der Ebene als im Gebirge; bis zum Meer), Argya Acaciae (in kleinen Gesellschaften bis nahe bei Sanakin), Passer simplex in Truppen bis 20 Stück an den Wüstenbrunnen ostwärts bis Ranai, Caprimulgus infuscatus überall einzeln, Pyrrhalauda crucigera (die afric. Varietat oder Art) in Paaren bis zum rothen Meer, Pterocles guttatus. Turtur semitorquatus einzelner, häufiger $T$. vinaceus und aegyptiacus. Alle bis zum rothen Meer. - Oena capensis ebenfalls, aber in sehr geringer Anzahl. Cercotrichas erythropterus überall, ebenso Lanius algeriensis. Am Djebel Erémid Vultur occipitulis. 
44 v. Heuglin: Vogelzug und Vorkommnisse in N.-O.-Afriea.

Alauda cristata ziemlich selten; häufiger am rothen Meer. Am Bir el Bak Vultur auricularis.

, In den Thälern von Ranai viele Sylvien, S. crassirostris, S. hypolais, S. garrula und wahrscheinlich S. fitis und melanocephala. Saxicola melanura bis gegen das rothe Meer.

In den Gebirgen von Abadab und Abu-Goloda Wachtel, Pirol und Blauracken in Menge, Flüge von Emberiza striolata, Lamprotornis chrysogaster; Uroloncha cantans. Einzelner Nectarinia gularis und eine zweite, kleine kurzschnäblige Art, von der ich nur das Jugendkleid sah. Ixos Arsinoe von Abadab bis zum rothen Meer.

Lanius cruentatus in Paaren.

Colius senegalensis vom Abadab östlich in Flügen bis auf $5000^{\prime}$ Meereshöhe. Saxicola deserti eben so, sowie S. sordida.

Micropogon margaritatus und wahrscheinlich Tockus erythrorhynchus am Abu-Goloda bis Wadi-Okuak. Hier fand ich auch Falco polyzonus und Textor galbula. Beide mit Nestbau beschäftigt.

Pterocles quadricinctus in Paaren in waldigen Gebirgsgegenden.

Otis arabs einzeln in grasreichen Thälern, wohl auf der ganzen Wegstrecke. Sehr merkwürdig für die Ornis Africa's ist aber das Factum, dass ich am Ostrand der Gebirge von Okuak einen Flug von Steinhühnern antraf, von denen ich ein Weibchen erlegte. Die Art scheint unbedingt der westasiatischen Perdix Hayii Temm. anzugehören, die ich eher zu Chacura als zu Ptilopachys stellen möchte. Sie ist in ihrem Benehmen, Ruf und Lebensweise ein wahres Steinhuhn, auch erzählte mir ein Araber, dass die Eier Aehnlichkeit mit Wachteleiern haben, also wohl gefleckt sind, was bei Ptilopachys nicht der Fall ist. - In der genannten Localität traf ich endlich Saxicola cachinnans und Pterocles Lichtensteinii; bei Sanakin Corvus curvirostris.

Natürlich ist bei meiner flüchtigen Durchreise hier wohl Vieles von mir übersehen worden; namentlich von kleinern Vögeln, wie Sylvien, Ammern etc. fiel mir Manches auf, das nicht erlegt werden konnte.

Für Merops albicollis, Colius senegalensis, Textor galbula, Nectarinia gularis, Micropogon margaritatus, Lamprotornis chrysogaster und Pterocles quadricinctus und Turtur vinaceus, wohl auch T. $l u$ gens und Lanius cruentatus mögen die bezeichneten Localitäten als nördlichste Gränze ihres Vorkommens in N.-O.-Africa gelten.

In den Gebirgen zwischen Ranai und Sanakin tritt die Regenzeit sehr spät ein, gewöhnlich erst mit Anfang August, wesshalb 
auch die Mauser erst um diese Periode erfolgt, sowie - wie schon bemerkt - einige Vogelarten erst Mitte September den Nestbau beginnen, während sämmtliche Sumpf- und Wasservögel auf den Inseln um Sanakin ihr Brutgeschäft längst beendigt haben.

Sanakin, 6. October $\mathbf{1 8 6 4 .}$

\section{Giterarische Berichte.}

\section{Katalog der Vögel Sardiniens mit Noten u. Beobachtungen von Tommaso Salvadori.}

Aus dem Italienischen übertragen durch

Dr. Carl Bolle.

[Mit mehren der hervorragendsten unter den naturwissenschaftlichen Erforschern Sardiniens durch zum Theil innige Bande verbunden, hat sich der Unterzeichnete, vermöge veröffentlichter und nicht veröffentlichter Mittheilungen, die Fauna-und Flora dieses interessanten Landes nahe gerückt gesehen. Er ergreift daher mit Vergnïgen die Gelegenheit, dem deutschen ornithologischen Publikum sowohl eine soeben erscheinende wichtige, Sardinien betreffende Arbeit im Gewande der vaterländischen Sprache vorzuführen, als auch dasselbe mit einem neuen und befähigten Ornithologen Italiens bekannt zu machen. Signore T. Salvadori hat seine Beobachtungen in den Mailänder „Atti della Società italiana di scienze naturali" vol. 6. niederzulegen begonnen. Das Interesse, welches dieselben für sich in Anspruch nehmen, dürfte vielleicht dadurch um so grösser werden, dass die Aussicht Dr. A. Hansmann's in Sardinien gemachte Wahrnehmungen ihrem ganzen Umfange nach publicirt zu-sehen, leider mehr und mehr schwindet.

Als Erinnerung an um Sardinien erworbene Verdienste, sowie als Tribut der Freundschaft, erlaubt es sich der Uebertrager die Namen de Notaris, Alfred Hansmann, Paul Ascherson und Otto Reinhardt an den Beginn seiner Arbeit zu setzen. Ein Lararium der Erforschung dieser Insel wird durch sie, wenn auch nicht vollständig, bezeichnet.

Berlin, am 31. October 1864.

C. Bolle.]

Sardinien ist sicher in Italien, vielleicht sogar in Europa, das in jeder Hinsicht wildreichste Land und sein Ruf in dieser Beziehung ist ein so anerkannter, dass alljährlich zahlreiche Gesell- 


\section{$2 \mathrm{BHL}$ Biodiversity Heritage Library}

Heuglin, Theodor von. 1865. "Notizen über den Vogelzug im Herbst 1864, sowie die ornithologischen Vorkommnisse in den Ländern der Bischárin, Omáráb und Hadendoa zwischen Berber und Sanakin." Journal $f u$

r Ornithologie 13, 42-45. https://doi.org/10.1007/bf02261152.

View This Item Online: https://www.biodiversitylibrary.org/item/109917

DOI: https://doi.org/10.1007/bf02261152

Permalink: https://www.biodiversitylibrary.org/partpdf/141966

\section{Holding Institution}

Smithsonian Libraries

\section{Sponsored by}

Biodiversity Heritage Library

\section{Copyright \& Reuse}

Copyright Status: Public domain. The BHL considers that this work is no longer under copyright protection.

This document was created from content at the Biodiversity Heritage Library, the world's largest open access digital library for biodiversity literature and archives. Visit BHL at https://www.biodiversitylibrary.org. 